\title{
Reducing Electron Beam Damage with Multipass Transmission Electron Microscopy
}

Colin Ophus ${ }^{1}$, Thomas Juffmann ${ }^{2}$, Stewart A Koppell ${ }^{2}$, Brannon B Klopfer ${ }^{2}$, Robert Glaeser ${ }^{3}$ and Mark A Kasevich ${ }^{2}$

1. National Center for Electron Microscopy, Molecular Foundry, Lawrence Berkeley National Laboratory, Berkeley, USA.

2. Department of Physics, Stanford University, Stanford, USA.

3. Molecular Biophysics and Integrative Bioimaging, Lawrence Berkeley National Laboratory, Berkeley, USA.

With the introduction of hardware aberration correction, direct electron detectors, ultra-bright electron sources and highly precise spectrometers, it seems like we are approaching the pinnacle of transmission electron microscopy (TEM) imaging and spectroscopy. However, the field of electron microscopy is still far from the ultimate signal-to-noise and efficiency limits imposed by electron scattering physics. Current TEM experiments where images are measured with high quantum efficiency detectors can approach the shot noise (or Poisson noise) limits, but the true upper bound for signal-to-noise for any electron imaging measurement is the Heisenburg limit [1]. Some initially proposed methods for approaching this measurement limit were quantum techniques such as highly-entangled measurement particles, which are impractical to use in a large-scale imaging experiments using photons. Entangling large numbers of electrons is even more difficult and seems unlikely to be achieved with existing technology. However, there is an alternative method to approach the Heisenburg limit: allowing the electron probe to interact with the sample many times before the image intensity measurement [2], as in the recently proposed design for a multipass transmission electron microscope [3,4].

In this talk, we will describe a multipass TEM instrument designed using the principles of quantum metrology. A simplified schematic of the experimental geometry is shown in Figure 1. A multipass TEM is very similar to a conventional TEM, except for the addition of two electron mirrors and the use of a pulsed electron source. The biasing of these electron mirrors can be reduced to allow the pulsed electron beam to enter the center portion of the instrument. The central portion is composed of two mirror-image configurations of a TEM instrument with mirrors on each end. The electron beam can therefore be passed through the sample multiple times. After a set number of passes, the electron beam can be passed out through the second mirror shown in the Figure 1 schematic. Finally, the electron pulse can be imaged using a standard electron detector, either after using a phase plate or applying a defocus to generate a phase contrast image. We assume that the mirrors and lenses can compensate for each other's aberrations in this configuration.

The advantage of using a multipass instrument is demonstrated in Figure 2. In this example, we have used multislice simulations to show how a multipass instrument could transform a low-dose imaging experiment of an isolated, small protein sample that is representative of cryo-EM experiments, the hexameric unit of the immature HIV-1 Gag CTD-SP1 lattice [5]. The structure and projected potential of this protein sample is shown in two different orientations in Figures 2a and b. Simulated TEM images at different electron doses for different numbers of passes are plotted in Figure 2c. The reported dose is the total dose seen by the specimen, which is approximately equal to one divided by the number of passes. These simulations show that with an increasing number of passes, the signal-to-noise increase up to 
approximately 16 passes. At higher numbers of passes, the signal-to-noise begins to fall due to phase wrapping, or inelastic losses, or both. In a multipass instrument, if the image is formed with $m$ passes, the total dose can be reduced by a factor of $m$ to generate an image with the same signal-to-noise. This relationship makes the multipass imaging protocol a promising method for dramatically reducing the required electron dose for imaging experiments in transmission electron microscopy [6].

\section{References:}

[1] V Giovannetti, S Lloyd, and L Maccone, Science 19 (2004), p. 1330.

[2] V Giovannetti, S Lloyd, and L Maccone, Physical Review Letters 96 (2006), p. 010401.

[3] P Kruit et al, Ultramicroscopy 164 (2016), p. 32.

[4] T Juffmann et al, Multi-pass Transmission Electron Microscopy (2016).

[5] JM Wagner et al, eLife 5 (2016), e17063.

[6] Work at the Molecular Foundry was supported by the Office of Science, Office of Basic Energy Sciences, of the U.S. Department of Energy under Contract No. DE-AC02-05CH11231. This research was also supported by the Gordon and Betty Moore Foundation and the Stanford Graduate Fellowship.

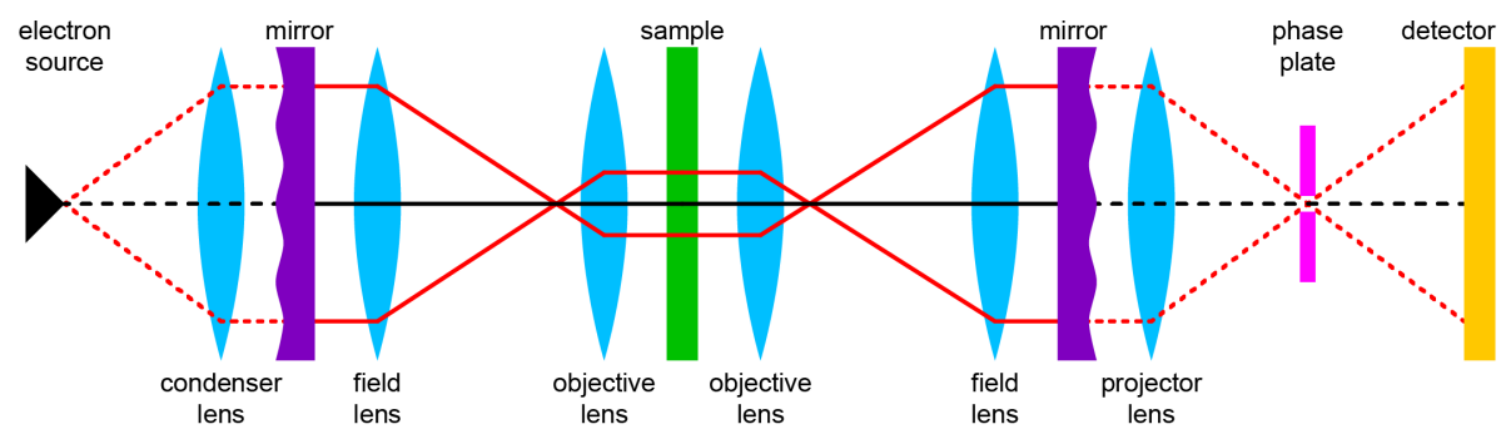

Figure 1. Simplified schematic of a multipass transmission electron microscope. Dashed lines show electron path traversed a single time, while solid lines show paths travelled multiple times.
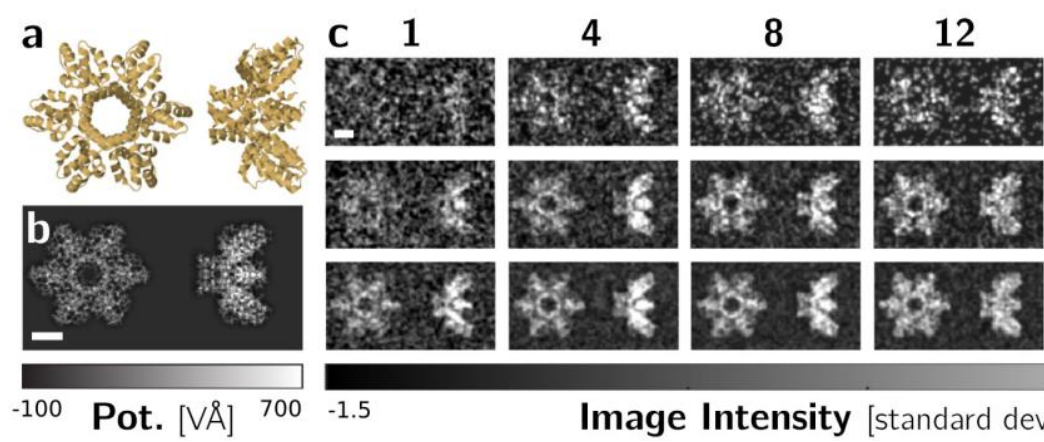

\section{6}

32 passes
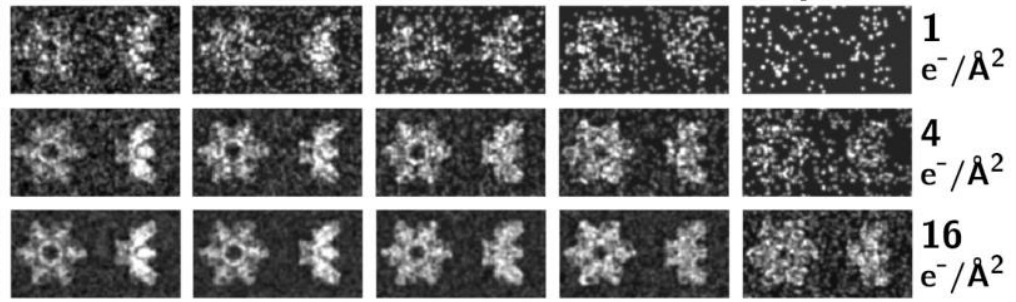

Image Intensity [standard deviations]

$$
4.5
$$

Figure 2. Multislice simulations of multipass TEM images of the HIV-1 Gag protein, taken from [4]. (a) Protein structure, and (b) projected potential view of protein from the top and side. (c) Images simulated with different numbers of passes and electron doses. Scalebars are $2 \mathrm{~nm}$. 